\title{
Dolor torácico agudo: Disección Aórtica
}

\author{
Yovana García Rioja ${ }^{a}$
}

a Médico de Familia. Centro de Salud Cabanillas del Campo (Guadalajara, España)

\section{Correspondencia:}

Yovana García Rioja.

Correo electrónico:

yobanag@sescam.jccm.es

Recibido el 22 de mayo de 2010.

Aceptado para su publicación el 17 de agosto de 2010.

\section{RESUMEN}

La disección aórtica consiste en la ruptura de la capa íntima de la pared del vaso con la formación de una falsa luz entre ésta y la capa media. Se trata de una emergencia vital con una elevada tasa de morbilidad y mortalidad y cuyo síntoma inicial más común es el dolor torácico agudo. Su diagnóstico precoz, a pesar de los avances en las pruebas complementarias, sigue representando un reto clínico.

Palabras Clave. Enfermedades de la Aorta, Dolor Torácico.

\section{ABSTRACT}

\section{Acute Chest Pain: Aortic Dissection}

Aortic dissection is a tear in the intimal layer of the vessel wall followed by the formation of a false lumen between this layer and the media layer. It is a life-threatening emergency with a high morbidity and mortality rate. The most common initial symptom is acute chest pain. Early diagnosis, in spite of advances in complementary tests, still remains a clinical challenge.

Key words. Aortic Diseases, Chest Pain.

\section{INTRODUCCIÓN}

El dolor torácico agudo es una de las causas más frecuentes de consulta en los servicios de urgencia extrahospitalarios ${ }^{1}$. Su importancia para el profesional radica en que es necesaria una orientación diagnóstica rápida que permita diferenciar aquél que indica enfermedad grave y que puede requerir un tratamiento médico o quirúrgico urgente, de aquél que traduce un proceso banal ${ }^{2}$.

A pesar de que la disección aórtica aguda no es una patología común, su gravedad hace imprescindible mantener un alto índice de sospecha. El paciente típico es un varón entre la $6^{\mathrm{a}}-7^{\mathrm{a}}$ década de la vida, con antecedentes de hipertensión arterial (factor clave), que presenta de forma súbita un dolor torácico severo de localización retroesternal o interescapular que se irradia hacia la espalda y abdomen ${ }^{3}$.

\section{OBSERVACIONES CLÍNICAS}

Varón de 65 años, fumador de 40 paquetes-año, con antecedentes de hipertensión arterial, síndrome de Apnea-Hipopnea del sueño grave e hipertrofia prostática benigna. En tratamiento con irbesartan-hidroclorotiazida, tamsulosina y CPAP a $8 \mathrm{~cm}$ de $\mathrm{H} 2 \mathrm{O}$ durante la noche. 
Acude al servicio de Urgencias por notar de forma repentina, y mientras se encontraba en reposo, dolor en región precordial y epigastrio que refiere como opresivo, sin cortejo vegetativo y que se irradia hacia la espalda cuando está tumbado, mejorando con la sedestación.

Exploración física: TA 200/98, SO2 97\%, auscultación cardiaca rítmica, sin soplos ni extratonos, auscultación pulmonar con disminución global del murmullo vesicular y roncus dispersos, adomen doloroso a la palpación en epigastrio, no masas ni megalias, no peritonismo, y sin signos de focalidad neurológica.

Pruebas complementarias: hemograma y bioquímica dentro de parámetros normales, ECG con ritmo sinusal a $50 \mathrm{lpm}$ y sin alteraciones agudas de la repolarización. En la figura 1 se muestran los hallazgos de la Rx tórax.

Ante los resultados de analítica, ECG y Rx y la discreta mejoría del paciente tras la administración de analgésicos y omeprazol, se decide su alta hospitalaria, siendo diagnosticado de epigastralgia inespecífica.

A las 24 horas somos requeridos para valorar al paciente en su domicilio, ya que presenta de nuevo dolor de similares características, destacando a la exploración marcada palidez cutáneo-mucosa, sudoracion e hipotensión (TA 90/50). Se deriva al nivel hospitalario, donde se le practica analítica en la que destacan: hemoglobina $9,6 \mathrm{mg} / \mathrm{dl}$, leucocitos 19000 , plaquetas 85000 y creatinina 2,2. ECG: ritmo sinusal, sin cambios respecto al del día previo.

En la figura 2 se muestran los hallazgos de la Rx tórax, ante los cuales se decide realizar TAC tóracoabdóminopélvico, donde se diagnostica una disección aórtica aguda tipo B de Stanford / III de De Bakey (figura 3). El paciente fue intervenido quirúrgicamente mediante colocación de endoprótesis aórtica y posterior drenaje de hemotórax con evolución favorable durante su estancia en $\mathrm{UCl}$ y en planta, por lo que fue dado de alta hospitalaria siguiendo actualmente controles periódicos.

\section{COMENTARIOS}

La incidencia de la disección aórtica no es bien conocida, estimándose entre 5-10 casos por millón de habitante y año. En su producción influyen dos circunstancias: una disminución de la resistencia de la pared arterial y un factor desencadenante, por lo que la incidencia se relacionará con la prevalencia de factores de riesgo como la hipertensión arterial, que es el factor desencadenante mas influyente, la edad y alteraciones en la pared aórtica ${ }^{4,5}$.

Existen dos clasificaciones para la disección aórtica en base a la localización anatómica: la de DeBakey y la de Stanford.

La clasificación de DeBakey incluye tres tipos:

- I: cuando se localiza en aorta ascendente, arco y aorta descendente.

- II: si se limita a la aorta ascendente.

- Illa: si se localiza tan sólo en aorta torácica descendente.

- IIlb: si se extiende desde la aorta torácica descendente a la aorta abdominal y arterias ilíacas.

La clasificación de Stanford reconoce dos tipos:

- A (proximal o ascendente): con extensión o no al arco y aorta descendente y que equivaldría a los tipos I y II de De Bakey.

- B: cuando se afecta exclusivamente la aorta descendente, correspondiendo a la III de DeBakey .

También se puede clasificar de acuerdo a la duración de los síntomas en aguda, si éstos están presentes durante menos de dos semanas (período de mayor morbi-mortalidad), y crónica, si duran más de ese tiempo ${ }^{3}$.

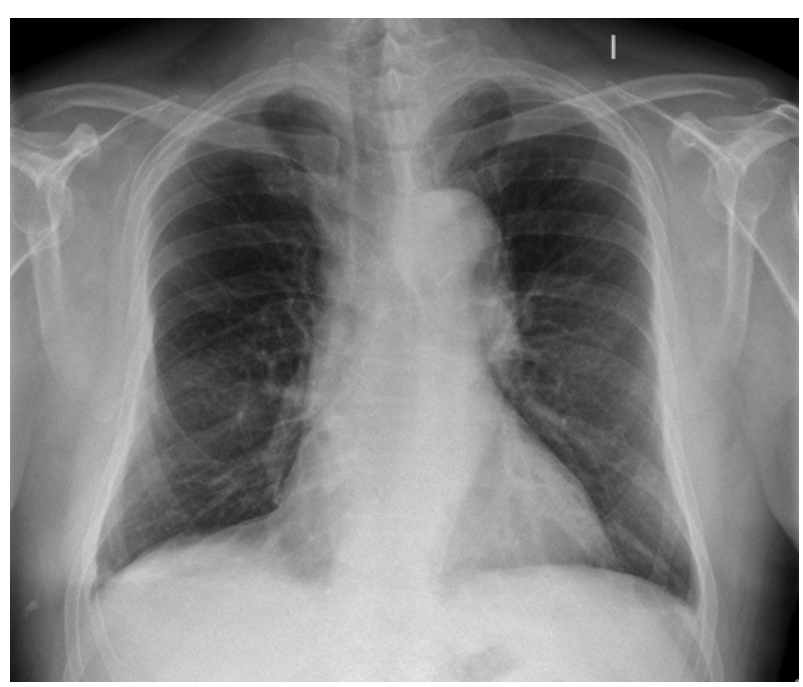

Figura 1. Primera visita a urgencias. Rx PA de tórax sin hallazgos de interés. 


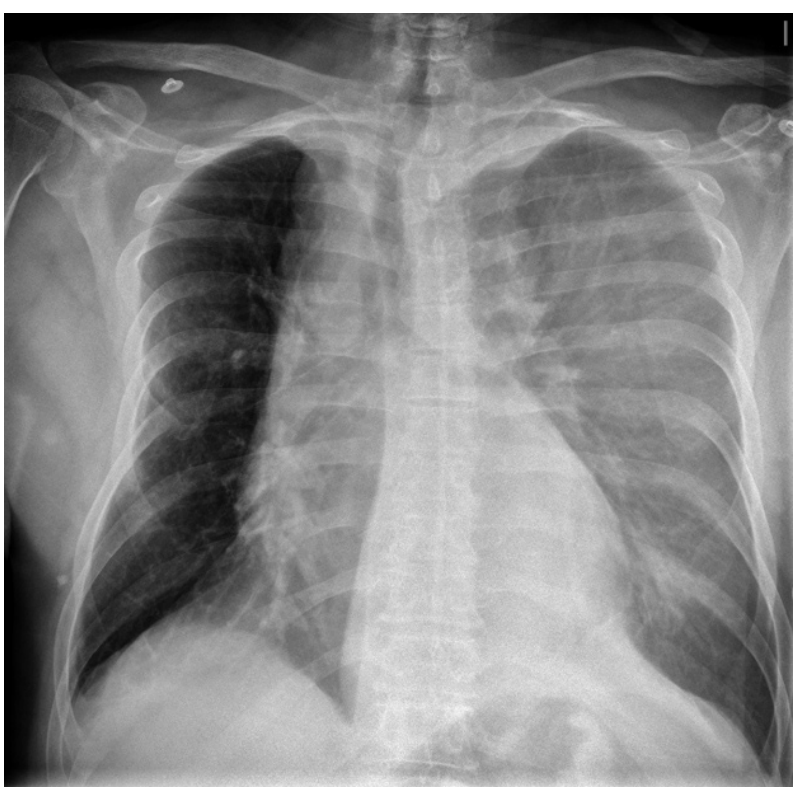

Figura 2. Rx PA de tórax ( $24 \mathrm{~h}$ después) con aumento de densidad difuso del hemitórax izquierdo con desplazamiento contralateral del mediastino y borramiento de la línea paraórtica.

La mortalidad de la disección aórtica sin tratamiento es altísima. Según la mayoría de los autores, más de un tercio de los pacientes mueren en la primeras 24 horas, la mitad en las siguientes 48 horas, dos tercios en la primera semana y casi el $90 \%$ muere en el primer mes ${ }^{7}$.

En la consulta de atención primaria o en los servicios de urgencia extrahospitalaria la valoración de un paciente con dolor torácico agudo supone un reto diagnóstico debido a que las pruebas complementarias disponibles son limitadas. Por ello, las herramientas fundamentales son la historia clínica y la exploración física, y basándonos en ellas deberemos hacer un despistaje precoz de aquellas patologías que puedan comprometer la vida del paciente ${ }^{8}$.

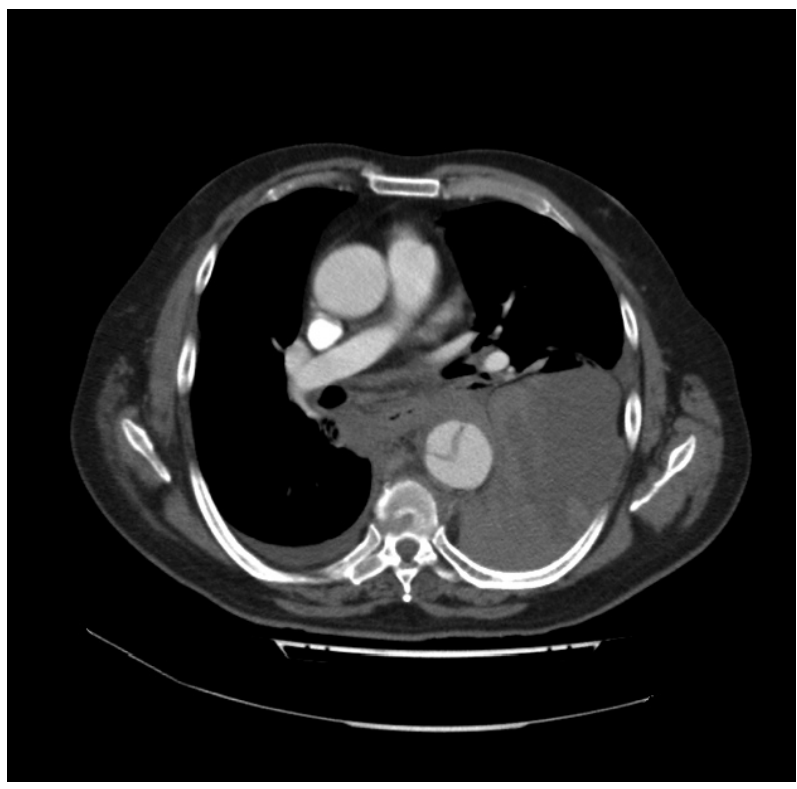

Figura 3. TC tórácica con contraste donde se evidencia un "flap" intimal en la aorta descendente, asi como derrame pleural izquierdo y hemomediastino.

\section{BIBLIOGRAFÍA}

1. Bayón Fernández J, Alegría Ezquerra E, Bosch Genover X, Cabadés, O'Callaghan A, Iglesias Gárriz I et al. Grupo de Trabajo ad hoc de la Sección de Cardiopatía Isquémica. Unidades de dolor torácico. Organización y protocolo para el diagnóstico de los síndromes coronarios agudos y Unidades Coronarias de la Sociedad Española de Cardiología. Rev Esp Cardiol. 2002; 55(2):143-54.

2. García Tejada J, Pindado Rodríguez C, Sanz Salvo J. El Dolor Torácico. En: Zarco Montejo J, Sáenz de la Calzada C, eds. Urgencias Cardiológicas. Madrid: Editorial Luzán; 2000. p. 7-24.

3. Prêtre R, Von Segesser LK. Aortic dissection. Lancet. 1997; 349:1461-4.

4. Muiño Miguez A, Villalba García MV, López GonzálezCobos C, Gómez Antúnez M, Ortiz Vega M, Ortiz Alonso J. Acute aortic dissection. An Med Interna (Madrid). 2002; 19(4):19-23.

5. Robicsek F, Thubrikar MJ. Hemodynamic considerations regarding the mechanism and prevention of aortic dissection .Ann Thorac Surg. 1994; 58:1247-53.

6. Daily PO, Truebold HW, Stinson EB, Wuerflein RD, Shumway NE. Management of acute aortic dissections. Ann Thorac Surg. 1970; 10:237-47.

7. Della H. Aortic dissection. RN. 2007; 70(3):26-31.

8. Muñoz Avila JA, Torres Murillo JM, Calderón de la Barca Gázquez JM, García Criado El, Jiménez Murillo LM.

Manejo del paciente con dolor torácico agudo en Atención Primaria. Semergen. 24(2):137-45. 